\title{
ORTHODONTIC TREATMENT WITH Nd-Fe-B MAGNETS
}

\author{
Valerii Kutsevlyak \\ Department of Orthopedic Dentistry No. $2^{1}$ \\ Vladyslav Starikov \\ Department of Pediatric Dentistry, Orthodontics and Implantology ${ }^{1}$ \\ doctordentistortho@gmail.com \\ Volodymyr Samofalov \\ Department of Metals and Semiconductors Physics ${ }^{2}$ \\ Vadym Starikov \\ Department of Technical Cryophysics ${ }^{2}$ \\ ${ }^{1}$ Kharkiv Medical Academy of Postgraduate Education \\ 58 Amosova str., Kharkiv, Ukraine, 61176 \\ ${ }^{2}$ National Technical University "Kharkiv Polytechnic Institute" \\ 2 Kyrpychova str., Kharkiv, Ukraine, 61002
}

\begin{abstract}
The aim. The development of methodology for experimental and theoretical assessment of interaction forces between magnets in an orthodontic apparatus, the test of corrosion resistance of protective oxide and nitride coatings deposited on $\mathrm{Nd}-\mathrm{Fe}-\mathrm{B}$ magnets surface.

Materials and methods. The Nd-Fe-B permanent magnets with saturation magnetization $\mathrm{M}_{\mathrm{s}} \approx 1100 \mathrm{G}$ and bilayer $\mathrm{ZrN} / \mathrm{ZrO}_{2}$ coatings were used. To experimental measure of interaction forces between magnets the device was assembled on the base of analytical balance. The distance between the magnets was varied using non-magnetic plates. The $\mathrm{ZrO}$, and $\mathrm{ZrN}$ coatings have been analyzed for their corrosion properties in $0.9 \% \mathrm{NaCl}$ quasi-physiological solution.

Results. An original method was proposed for calculating of magnetic interaction forces for materials with high magnetic anisotropy, which has good agreement with experimental measurement of forces. The theoretical model takes into account the size of the magnets and the mutual influence of their opposite faces. An increase of corrosion resistance of magnetic materials can be provided by zirconium oxide or nitride compounds, which contribute to inhibition of electrochemical corrosion of Nd-Fe-B magnets.

Conclusions. A method for calculating of interaction forces between permanent magnets, which are used for correction of malocclusion in orthodontic, has been developed. The passivation of the Nd-Fe-B permanent magnets surface can be achieved by applying of bilayer $\mathrm{ZrN} / \mathrm{ZrO}_{2}$ coating.
\end{abstract}

Keywords: malocclusion, tooth movement, Nd-Fe-B magnets, magnetic interaction forces, corrosion resistance, coating.

DOI: $10.21303 / 2504-5679.2020 .001316$

\section{Introduction}

Anomalies in dentition structure require orthodontic treatment to achieve a higher aesthetics and functionality. Correction of bite pathologies is carried out using bracket systems in most cases $[1,2]$. This treatment is long and requires careful observance of oral hygiene. However, there is another way to correct orthodontic pathologies - the use of devices with magnets [3, 4]. Such devices have several advantages: mechanical transfer of force without friction; predictable magnitude of force; high stability of parameters of modern magnetic materials; easier tooth brushing due to a significant simplification of the device design [5]. In addition, the magnetic stimulation can significantly reduce the duration of orthodontic treatment $[6,7]$.

For the first time, magnets in dentistry began to be used more than 70 years ago $[8,9]$. They were made of Al-Ni-Co or Pt-Co alloys and were used as fixing elements for partial and complete removable dentures [10]. Such magnets provided a low magnitude of magnetic interaction force, which led to necessity of their size increase. Because of this, their use in orthodontics was difficult $[11,12]$. 
Currently, magnets containing rare earth metals are interesting, for example, Nd-Fe-B. Investigation of these magnets have shown the absence of any negative effect of magnetic field on the body $[13,14]$. Such magnets demonstrate improved properties compared to their predecessors [15] and provide a significantly higher magnitude of magnetic forces. This allows to use of small magnets comparable to the dimensions of braces.

Permanent magnets in attractive or repulsive configurations have a continuous effect on each other [16]. Based on this, an accurate assessment of magnets interaction force is necessary both to prevent negative side effects at exposure of excessive forces, and to determine the initial position of magnets in relation to each other. But known calculation methods [17, 18] are lengthy and do not always provide the necessary calculations accuracy.

A significant drawback of magnetic materials is their low corrosion resistance, which leads to destruction of the magnet during electrochemical corrosion in an aggressive environment of the oral cavity [19]. The situation can be corrected by applying various types of protective coatings, leading to passivation of magnetic material surface. Such coatings can be oxide or nitride films on the base of valve materials [20,21]. However, currently there is a lack of information on passivation methods of magnets surface used in orthodontic appliances, and the characteristics of the effectiveness of protective coatings.

The aim of work was the development of methodology for experimental and theoretical assessment of the interaction forces between magnets in an orthodontic apparatus, as well as to test the corrosion resistance of protective oxide and nitride coatings deposited on the surface of Nd-Fe-B magnets.

\section{Materials and methods}

\section{1. Materials}

We used Nd-Fe-B permanent magnets in the form of rectangular parallelepipeds with sizes of $4 \times 2 \times 4 ; 5 \times 2 \times 5$ and $6 \times 2 \times 6 \mathrm{~mm}$. Magnets manufactured by the company Polus-N (Kharkiv, Ukraine) which specializes in production of magnetic materials on the base of rare-earth alloys. Magnet saturation magnetization was $M_{s} \approx 1100 \mathrm{G}$ and maximum specific energy of magnets $W_{\max }=5.2 \times 10^{7} \mathrm{G} \cdot \mathrm{Oe}$.

To increase corrosion resistance of magnets, the oxide and nitride protective coatings were applied to their surface. The $\mathrm{ZrO}_{2}$ and $\mathrm{ZrN}$ coatings on $\mathrm{Nd}-\mathrm{Fe}-\mathrm{B}$ magnets were deposited in Bulat- 6 type device by condensing vacuum-arc plasma purified from macro-particulates using the curvilinear filter [20]. The magnets were fixed in the holder that squeezed its opposite sides with a small effort. Chemically pure zirconium (99.999) was used as a cathode material. The chamber was preliminary pumped out to a pressure of $6 \times 10^{-5}$ Torr.

At $\mathrm{ZrO}_{2}$ coating forming the pulsed negative bias of $1000 \mathrm{~V}$ with frequency $50 \mathrm{kHz}$ was applied to the sample holder from the source. The rotation of the holder was turned on, vacuum arc was ignited $\left(I_{d}=115 \mathrm{~A}\right)$ and the four faces of the magnets were cleaned by zirconium ions in the pulsed mode: cleaning of $1.5 \mathrm{~s}$ and pause of $6 \mathrm{~s}$; in total 15 cycles. Then the source was turned off, the chamber was filled with oxygen to a pressure of about $4 \times 10^{-3}$ Torr and zirconium dioxide was deposited during 12 minutes. Then the vacuum chamber was opened, the magnets were installed in the other position and $\mathrm{ZrO}_{2}$ was deposited on uncoated faces for 7 minutes.

At $\mathrm{ZrN}$ coatings deposition the bias potential was applied to the substrate from RF generator, which produced oscillations impulses of $5 \mathrm{MHz}$ frequency. Nitrogen $99.999 \%$ purity was used as an active gas. The $\mathrm{Zr}$ buffer layer of $20 \mathrm{~nm}$ thickness was deposited before the nitride coatings to improve its adhesion. Deposition of $\mathrm{ZrN}$ carried out at pressure $P=5 \times 10^{-4} \mathrm{~Pa}$ during $25 \mathrm{~min}$.

\section{2. Experimental procedures}

Scheme of magnets mutual arrangement, which was used in the calculations and experimental measurements of attractive force, is shown in Fig. 1. The magnets were located at distance $L$ from each other so that the vectors of their magnetizations were aligned with the easy magnetization axis and provide maximum strength for their interaction. 


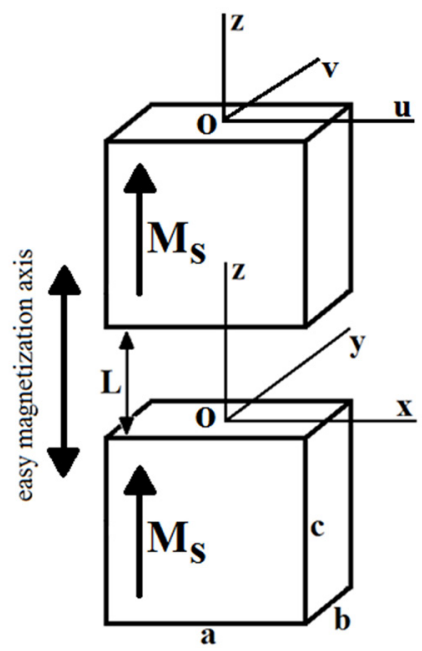

Fig. 1. The relative position of magnets at experimental measurement of their interaction strength and designations used in theoretical calculations.

According to experimental data, the force magnitude required for teeth body movement is in the range of 20-300 g [22]. Diagram of experimental plant to measure the interaction forces between magnets is shown in Fig. 2.

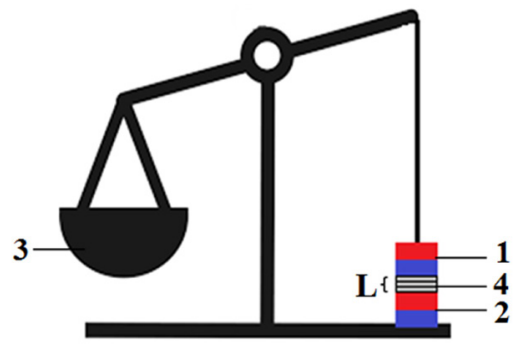

Fig. 2. Plant to experimental measure of interaction forces between magnets: 1, 2 - magnets; 3 - scale; 4 - set of non-magnetic spacers.

The plant was assembled on the base of analytical balance and included two magnets, one of which (1) was attached to the balance arm and the other one (2) - to the balance base. When weights were placed in the scale (3) there was a force that separated the magnets from each other. The load magnitude when the upper magnet was torn off from the lower was taken as the force of their attraction. The measurement took into account the weight of the upper magnet. Each measurement was repeated at least 30 times for necessary statistics. The distance L between the magnets was varied using a set of flat non-magnetic plates (4) $1.4 \mathrm{~mm}$ thick. The plant ensured the accuracy of measurement $\pm 2 \mathrm{~g}$.

The $\mathrm{ZrO}_{2}$ and $\mathrm{ZrN}$ coatings have also been analyzed for their corrosion properties in $0.9 \%$ $\mathrm{NaCl}$ quasi-physiological solution [21].

\section{Results}

The determination of interaction forces between the magnets was carried out both theoretically and experimentally at various distances $L$ between the magnets.

Theoretical calculations made it possible to determine the optimal minimum size of magnets to achieve a given attractive force. The interaction force between the magnets is defined as a gradient of their magnetostatic interaction energy $W$ :

$$
\vec{F}=-\nabla W
$$


The energy density of interaction between the magnets is equal to [9]:

$$
W=-(\vec{M} \times \vec{H}),
$$

where $\vec{M}$ is the magnetic moment of the first magnet; $\vec{H}$ is the scattering magnetic field created by the second magnet in the volume of the first magnet.

The calculation of the force $\vec{F}$ from equations (1) and (2) is possible if the direction of magnetization $\vec{M}$ and the scattering field strength $\vec{H}$ at each point of the magnets are known. The solution to this complex variational problem is achieved only by numerical methods. However, the determination of an interaction force between magnets with very large field of uniaxial anisotropy $H_{k}$ is significantly simplified. So, if the anisotropy field $H_{k}>100 \mathrm{Oe}$, the magnetization vector $\vec{M}$ is parallel to the easy magnetization axis at all points of magnet. Such large anisotropy occurs in compounds of transition metals Fe, Co with some rare-earth elements, as well as in ordered alloys Fe-Pt, Co-Pt. The arising scattering fields $H_{s}$ are small in comparison with the field $H_{k}$ and therefore practically do not change the direction of magnetization and the magnetization is distributed almost uniformly over the volume of magnet. The sources of scattering fields are "magnetic charges" that arise on surfaces where $\operatorname{div} \vec{M} \neq 0$. As a result, the calculation of interaction forces is reduced to solving the problems of electrostatics.

For magnets system in Fig. 1, "magnetic charges" with a density $\sigma= \pm M_{s}$ - saturation magnetization of magnet material) arise on the faces of the parallelepiped which are parallel to the XOY plane. The density of energy and interaction forces will be respectively equal to:

$$
\begin{gathered}
W=-(\vec{M} \times \vec{H}) \\
\vec{F}=\nabla\left(M_{Z} \times H_{Z}(x, y, z)\right)=M_{Z}\left(\frac{\partial H_{Z}}{\partial x} \vec{i}+\frac{\partial H_{Z}}{\partial y} \vec{j}+\frac{\partial H_{Z}}{\partial z} \vec{k}\right) .
\end{gathered}
$$

Since the magnet system used in the measurements, in equilibrium state

$$
\frac{\partial H_{Z}}{\partial x}=\frac{\partial H_{Z}}{\partial y}=0
$$

then

$$
F=F_{Z}(x, y, z)=M_{Z} \frac{\partial H_{Z}}{\partial z}
$$

Using equation (5), one can calculate the value of force for magnets of specific sizes. The component of scattering field $H_{z}$ created by the upper face of lower magnet at the point of upper magnet with coordinates $(u, v, z)$ is:

$$
H_{Z}=M_{S} \int_{-0,5 b-0,5 a}^{0,5 b} \int_{\left\{(x-u)^{2}+(y-v)^{2}+z^{2}\right\}^{\frac{3}{2}}}^{0,5 a} .
$$

$H_{z}$ component gradient of this field:

$$
\nabla H_{Z}=M_{S} \int_{-0,5 b}^{0,5 b} \int_{-0,5 a}^{0,5 a}\left[\frac{1}{\left\{(x-u)^{2}+(y-v)^{2}+z^{2}\right\}^{\frac{3}{2}}}-\frac{3 z^{2}}{\left\{(x-u)^{2}+(y-v)^{2}+z^{2}\right\}^{\frac{5}{2}}}\right] \mathrm{d} x \mathrm{~d} y .
$$

Substituting the equation for the gradient (7) into (5) we obtain for the force $F_{z}$ component associated with charges on the upper face of lower magnet following equation: 


$$
F_{Z}(L)=M_{S}^{2} \int_{L}^{L+c} \int_{-0,5 b}^{0,5 b} \int_{-0,5 a}^{0,5 a} \int_{-0,5 b}^{0,5 b} \int_{-0,5 a}^{0,5 a}\left[\frac{1}{\left\{(x-u)^{2}+(y-v)^{2}+z^{2}\right\}^{\frac{3}{2}}}-\frac{3 z^{2}}{\left\{(x-u)^{2}+(y-v)^{2}+z^{2}\right\}^{\frac{5}{2}}}\right] \mathrm{d} x \mathrm{~d} y \mathrm{~d} u \mathrm{~d} v \mathrm{~d} z .
$$

In the general case, the interaction force of magnets will be less than the value calculated by formula (8) due to the influence of lower face of lower magnet. This face interacts with the upper magnet oppositely to the action of lower magnet upper face, since the opposite faces of magnet have a magnetization divergence of different signs. For large magnets (c $>5 \mathrm{~mm}$ ) in the direction of $\mathrm{OZ}$ axis (Fig. 1) this factor can be neglected due to the fast attenuation of magnetic forces with distance. However, since it is advisable to use small magnets in orthodontics, which are comparable in size to locks of bracket systems, this factor should be taken into account. The calculation of the second component for interaction force, taking into account the sign of "magnetic charges" of lower face, is carried out according to formula (8) with modified limits for the first integral. Thus, the final equation for interaction forces between magnets located according to the scheme (Fig. 1) will be as follows:

$$
\begin{aligned}
& \left.F_{Z}(L)=M_{S}^{2} \int_{L}^{L+c} \int_{-0,5 b}^{0,5 b} \int_{-0,5 a}^{0,5 a} \int_{-0,5 b-0,5 a}^{0,5 b} \int_{-\frac{1}{2,5 a}\left[\frac{3 z^{2}}{\left\{(x-u)^{2}+(y-v)^{2}+z^{2}\right\}^{\frac{5}{2}}}\right.}\right] \mathrm{d} x \mathrm{~d} y \mathrm{~d} u \mathrm{~d} v \mathrm{~d} z- \\
& -M_{S}^{2} \int_{L+c}^{L+2 c} \int_{-0,5 b}^{0,5 b} \int_{-0,5 a}^{0,5 a} \int_{-0,5 b}^{0,5 b} \int_{-0,5 a}^{0,5 a}\left[\frac{1}{\left\{(x-u)^{2}+(y-v)^{2}+z^{2}\right\}^{\frac{3}{2}}}-\frac{3 z^{2}}{\left\{(x-u)^{2}+(y-v)^{2}+z^{2}\right\}^{\frac{5}{2}}}\right] \mathrm{d} x \mathrm{~d} y \mathrm{~d} u \mathrm{~d} v \mathrm{~d} z .
\end{aligned}
$$

Fig. 3 shows the calculated dependences of attractive forces for magnets of size $6 \times 2 \times 6$ $\mathrm{mm}$ both without and with taking into account the action of the lower face of lower magnet. As can be seen from the figure, both dependences practically coincide, therefore, the correction for calculations without taking into account the action of the lower face is insignificant in this case. If we reduce the size of the lower and upper magnets along $O Z$ axis, this correction will begin to grow (Fig. 4), reaching $40 \%$ at magnets thickness $2 \mathrm{~mm}$ and distance between magnets $2.8 \mathrm{~mm}$.

Comparison of calculated and experimentally measured interaction force values of magnets of size $5 \times 2 \times 5 \mathrm{~mm}$ is shown in Fig. 5 . The theoretical model adopted in the calculations gave a fairly accurate estimate of magnetic interaction forces (difference is not more than $10 \%$ ). Therefore, the proposed formulas allow in most cases to exclude the experimental assessment of the magnetic interaction forces and control it only periodically for estimate of main parameters of magnetic materials.

Analysis of jaw teeth sizes showed that the range of magnets that are promising for use in orthodontics could include three types of sizes $4 \times 2 \times 4 ; 5 \times 2 \times 5$ and $6 \times 2 \times 6 \mathrm{~mm}$. The calculated curves for each of these magnets types are shown in Fig. 6. The dotted line in the figure indicates the threshold distance between magnets, which provide beginning of teeth movement. As it can be seen, the range of distances for the most efficient use of Nd-Fe-B magnets is on average 2-7 mm.

Orthodontic treatment using Nd-Fe-B magnets in aggressive environment of oral cavity can cause their electrochemical corrosion, especially since the duration of such treatment is usually several months. To block of electrochemical corrosion processes the use of oxide or nitride coatings is proposed. Among coatings of these types the zirconium oxides and nitrides relatively high corrosion resistance [20,21]. For example, zirconium oxide is widely used in dentistry for production of non-metal ceramic prostheses [21]. The results of corrosion tests of magnets in the initial state and after deposition protective coatings are presented in Fig. 7. 


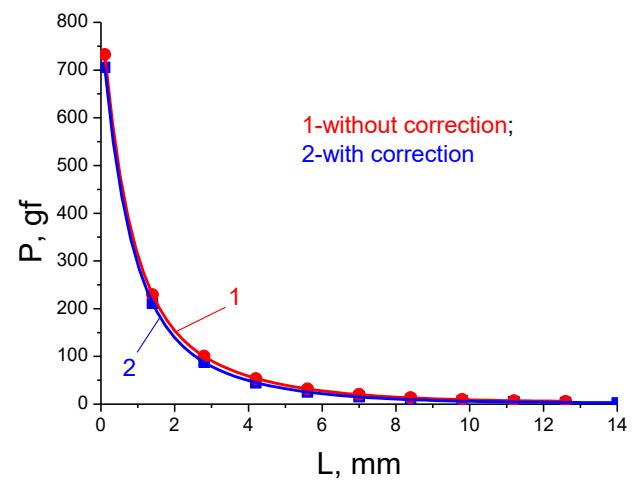

Fig. 3. Comparison of calculated dependences of magnets interaction forces on distance between them both without (1) and with (2) taking into account the action of the lower face

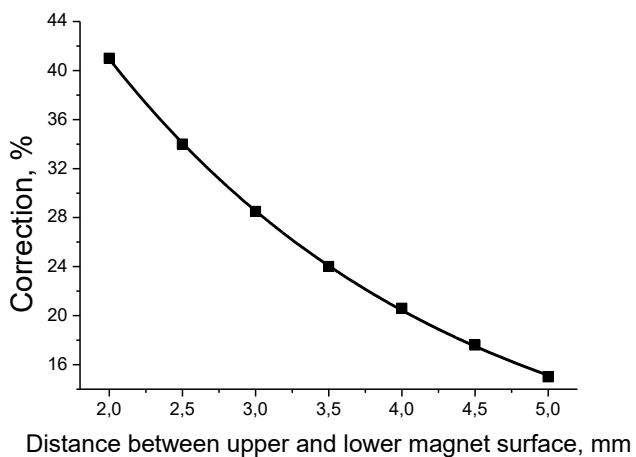

Fig. 4. Dependence of correction magnitude on the distance between the faces of magnets along $O Z$ axis

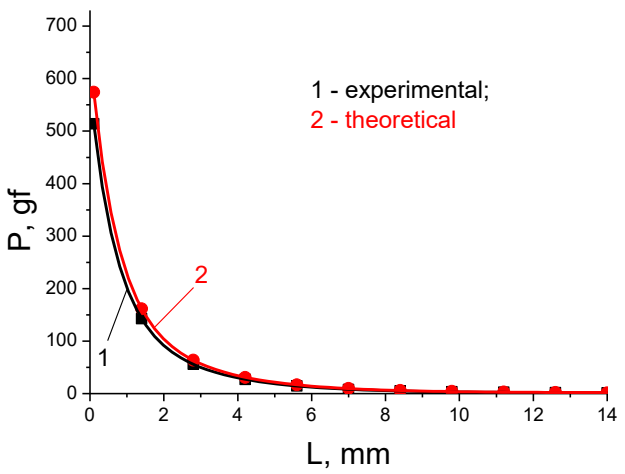

Fig. 5. Comparison of experimental (1) and theoretical (2) dependences of the interaction force between magnets $5 \times 2 \times 5 \mathrm{~mm}$

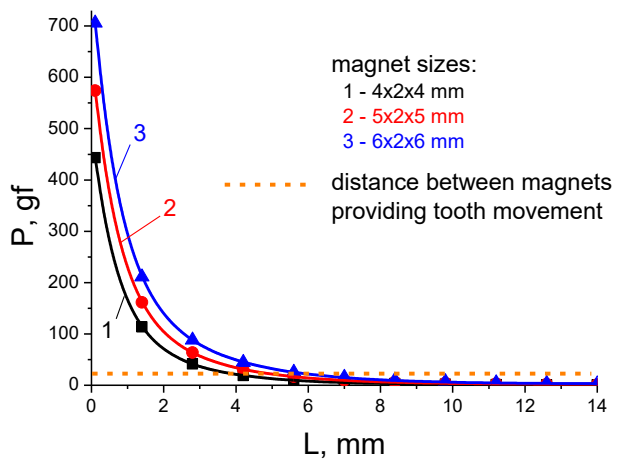

Fig. 6. Calculated dependences of interaction force between magnets for use in orthodontics 


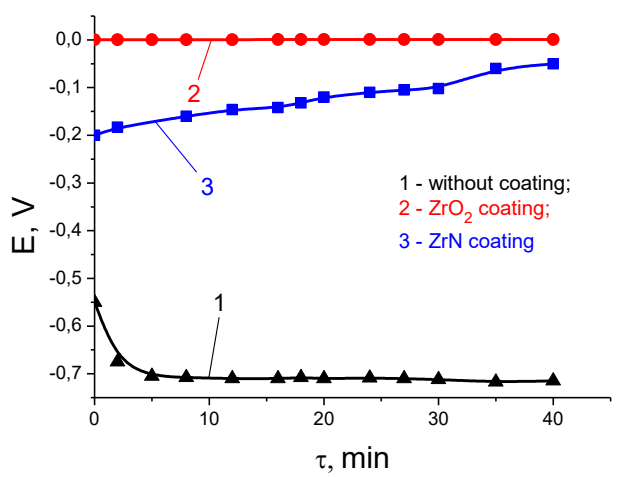

Fig. 7. Time dependence of the electrode potential of magnets in the initial state (1) and after deposition of oxide (2) or nitride (3) coatings

An uncoated magnet shows a rather low electrode potential (Fig. 7, curve 1), comparable with the potential of undoped iron-based alloys. In combination with other structural elements of the orthodontic apparatus (rings, crowns, guides), unprotected magnets will corrode and collapse quickly enough in the patient's mouth, which is unacceptable from both points of view: destruction of orthodontic apparatus and extraction of corrosion products that may cause toxic effect on the body.

Depositing of zirconium oxide with thickness up to $3 \mu \mathrm{m}$ significantly changes the situation (Fig. 7, curve 2). The electrode potential of the oxide-coated magnet has shifted to the region of positive values, reaching the level of electrode potential of zirconium oxide bulk sample. Since the tests were carried out in electrolytic aqueous solution, due to electroosmotic processes it was possible to control the coating submicroporosity, which would be problematic to detect in the usual way. The stability of electrode potential over time indicates the continuity of the oxide coating not only on the magnet planes, but also on its corners and edges. It is important that the magnet size has not critically changed due to the small thickness of the coating.

A similar situation, but to a lesser extent, is observed when applying a nitride coating (Fig. 7, curve 3). However, a significant advantage of the nitride coating is its high cosmetic effect. It, in contrast to the black oxide coating, has a pale yellow color, which is more acceptable for the patient. The less pronounced stability of the electrode potential of nitride coating, compared with the oxide one, may indicate a variation in its thickness and possible insignificant porosity. To achieve the maximum protective and cosmetic effect it is advisable to use bilayer coatings where the oxide layer provides high metal resistance against electrochemical corrosion and the nitride layer provides the magnet surface with a high cosmetic effect. Electrochemical tests showed that the electrode potential of such bilayer coating coincides in value with the oxide coating potential (Fig. 7, curve 2).

\section{Discussion}

The experimental data obtained in the work on determination of interaction forces between $\mathrm{Nd}-\mathrm{Fe}-\mathrm{B}$ magnets showed that modern materials science technologies make it possible to produce magnets of required size, comparable with the size of braces-locks, providing the necessary forces for various tooth movements for correcting of malocclusion. A universal method for calculating of permanent magnet interaction forces was proposed for the first time, determined by the shape and, consequently, the distribution of magnetic fields near the magnet surface. The calculating results of fields and interaction forces between the magnets indicate good agreement between the measured and calculated dependences (the difference is not more than $10 \%$ ). Until now $[4,18]$, the forces of magnetic interaction have been determined only experimentally, and theoretical calculations have not taken into account the effect of opposite magnets faces, acting in antiphase, and other factors of such interaction.

Because of low corrosion resistance, it is necessary passivation of the Nd-Fe-B permanent magnets surface. It can be achieved by applying of bilayer $\mathrm{ZrN} / \mathrm{ZrO}_{2}$ coating, where the oxide layer provides high resistance to electrochemical corrosion of metal alloy and the nitride layer provides 
the magnet surface with a high cosmetic effect. In most literary sources [19, 21], including similar studies, the formation of an effective continuous protective coating was not confirmed. Moreover, in view of certain technological difficulties, it was proposed to completely abandon the vacuum coating, and place the magnets in a tantalum foil capsule using laser welding.

Study limitations. As limitations to the use of magnets in orthodontic appliances, it should be noted that the calculation procedure could be significantly more complicated when using magnets have cylindrical or teardrop shape. In addition, the proposed technology for surface passivation is difficult to implement in the presence of holes in the magnet or the manufacture of complex magnets, when individual parts of the surface can be shaded at vacuum deposition of material, and the coating ceases to be continuous.

Prospects for further research. In the future, it is planned to develop a model that describes the interaction of several magnets mounted on a number of teeth of the dental arch.

Proposed method can be used to solve other problems of orthodontics, for example, to rotate teeth around its axis. In addition, theoretical calculations are especially necessary in cases where directly measure of interaction force for the orthodontic apparatus with magnets is impossible.

\section{Conclusions}

A method for calculating of interaction forces between permanent magnets, which are used for correction of malocclusion in orthodontic, has been developed. The passivation of the Nd-Fe-B permanent magnets surface can be achieved by applying of bilayer $\mathrm{ZrN} / \mathrm{ZrO}_{2}$ coating.

\section{Conflict of interest}

The authors declare there is no conflict of interest.

\section{References}

[1] Proffit, W., Fields, H. (2012). Contemporary Orthodontics. Elsevier, 262-372. Available at: https://www.elsevier.com/books/ contemporary-orthodontics/proffit/978-0-323-08317-1

[2] Wiechmann, D. (2003). A New Bracket System for Lingual Orthodontic Treatment. Journal of Orofacial Orthopedics, 64 (5), 372-388. doi: http://doi.org/10.1007/s00056-003-0325-4

[3] Prasad, M., Manoj-Kumar, M., Gowri-Sankar, S., Chaitanya, N., Vivek-Reddy, G., Venkatesh, N. (2016). Clinical evaluation of neodymium-iron-boron (Ne2Fe14B) rare earth magnets in the treatment of mid line diastemas. Journal of Clinical and Experimental Dentistry, 8, 164-171. doi: http://doi.org/10.4317/jced.52352

[4] Tomizuka, R., Kanetaka, H., Shimizu, Y., Suzuki, A., Igarashi, K., Mitani, H. (2006). Effects of Gradually Increasing Force Generated by Permanent Rare Earth Magnets for Orthodontic Tooth Movement. The Angle Orthodontist, 76 (6), $1004-1009$. doi: http://doi.org/10.2319/071805-237

[5] Li, L. C. F., Wong, R. W. K., King, N. M. (2008). Orthodontic traction of impacted canine using magnet: a case report. Cases Journal, 1 (1), 382. doi: http://doi.org/10.1186/1757-1626-1-382

[6] Yin, Z. P., Guo, L., Wang, T. Y., Lv, S. L., Che, Y. M. (2013). Effect of magnetic field on the proliferation of human periodontal ligament fibroblasts. Contemporary Medicine, 19, 10-11.

[7] Showkatbakhsh, R., Jamilian, A., Showkatbakhsh, M. (2010). The effect of pulsed electromagnetic fields on the acceleration of tooth movement. World Journal of Orthodontics, 11, 52-56. Available at: https://www.ncbi.nlm.nih.gov/pubmed/21490989

[8] Darendeliler, M. A. (2006). Use of Magnetic Forces in Growth Modification. Seminars in Orthodontics, 12 (1), $41-51$. doi: http://doi.org/10.1053/j.sodo.2005.10.008

[9] Ravindran, K. V. (2011). Role of magnets in orthodontics - a review. Indian Journal of Dentistry, 2 (4), 147-155. doi: http:// doi.org/10.1016/s0975-962x(11)60036-1

[10] Bhat, V., Shenoy, Kk., Premkumar, P. (2013). Magnets in dentistry. Archives of Medicine and Health Sciences, 1 (1), $73-79$. doi: http://doi.org/10.4103/2321-4848.113587

[11] Darendeliler, M. A., Darendeliler, A., Mandurino, M. (1997). Clinical application of magnets in orthodontics and biological implications: a review. The European Journal of Orthodontics, 19 (4), 431-442. doi: http://doi.org/10.1093/ejo/19.4.431

[12] Linder-Aronson, A., Lindskog, S., Rygh, P. (1992). Orthodontic magnets: effects on gingival epithelium and alveolar bone in monkeys. The European Journal of Orthodontics, 14 (4), 255-272. doi: http://doi.org/10.1093/ejo/14.4.255

[13] Singh, D., Jain, U., Prakash, A. (2016). Magnets in orthodontics. Journal of Applied Dental and Medical Sciences, 2 (2), 128-134. Available at: http://www.joadms.org/home/article_abstract/120 
[14] Bondemark, L., Kurol, J., Larsson, A. (1998). Long-term effect of orthodontic magnets on human buccal mucosa - a clinical, histological and immunohistochemical study. European Journal of Orthodontics, 20 (3), 211-218. doi: http://doi.org/10.1093/ ejo/20.3.211

[15] Sharma, N. S., Kamble, R., Shrivastav, S., Sharma, P. (2015). The Use of Magnets in Orthodontics. World Journal of Dentistry, 6 (1), 45-48. doi: http://doi.org/10.5005/jp-journals-10015-1311

[16] Samofalov, V. N., Ravlik, A. G., Belozorov, D. P., Avramenko, B. A. (2004). Generation of strong inhomogeneous stray fields by high-anisotropy permanent magnets. Journal of Magnetism and Magnetic Materials, 281 (2-3), 326-335. doi: http://doi.org/ 10.1016/j.jmmm.2004.04.122

[17] Samofalov, V. N., Belozorov, D. P., Ravlik, A. G. (2006). Optimization of systems of permanent magnets. The Physics of Metals and Metallography, 102 (5), 494-505. doi: http://doi.org/10.1134/s0031918x06110068

[18] Samofalov, V. N., Belozorov, D. P., Ravlik, A. G. (2013). Strong stray fields in systems of giant magnetic anisotropy magnets. Physics-Uspekhi, 56 (3), 269-288. doi: http://doi.org/10.3367/ufne.0183.201303e.0287

[19] Kitsugi, A., Okuno, O., Nakano, T., Hamanaka, H., Kuroda, T. (1992). The corrosion behavior of Nd2Fe14B and SmCo5 magnets. Dental Materials Journal, 11, 119-129. doi: http://doi.org/10.4012/dmj.11.119

[20] Taran, A., Garkusha, I., Taran, V., Muratov, R., Starikov, V., Baturin, A. et. al. (2018). Structure and properties of nanostructured $\mathrm{ZrN}$ coatings obtained by vacuum-arc evaporation using RF discharge. Nanotechnology Perceptions, 14 (3), $167-177$. doi: http://doi.org/10.4024/n15ta18a.ntp.14.03

[21] Starikov, V. V., Starikova, S. L., Mamalis, A. G., Lavrynenko, S. N. (2016). Features of medical implant passivation using anodic oxide films. Journal of Biological Physics and Chemistry, 16 (2), 90-94. doi: http://doi.org/10.4024/08st16a. jbpc. 16.02

[22] Bondemark, L. (2000). A comparative analysis of distal maxillary molar movement produced by a new lingual intra-arch Ni-Ti coil appliance and a magnetic appliance. The European Journal of Orthodontics, 22 (6), 683-695. doi: http://doi.org/10.1093/ ejo/22.6.683 